tive radius $r$. Let the center $x_{0}$ be the sequence $\left\{k_{i}^{0}\right\}$, and let $s$ be chosen so large that $2^{-s-1}+2^{-s-2}+\cdots<r$. Now a point $X \equiv\left\{j_{i}\right\}$ of $D$ exists such that $\lim _{n} f_{n}(X)=+\infty$, and such that $j_{s+1}>k_{s}^{0}$. If we define $x_{1}$ as $\left(k_{1}^{0}, k_{2}^{0}, \cdots, k_{s}^{0}, j_{s+1}, j_{s+2}, \cdots\right)$, then $x_{1}$ belongs to $K$ and $\lim _{n} f_{n}\left(x_{1}\right)=+\infty$. Consequently $x_{1}$ cannot be a point of $U_{\mu}$ and this contradiction establishes $U$ as a set of the first category.

In a similar fashion it may be shown that the set $V$ of all $x$ in $D$ for which $\lim \inf _{n} f_{n}(x)>-\infty$ is likewise a set of the first category. Hence if we set $W \equiv U+V$ the theorem follows.

Finally, let $\sum u_{k}$ be a convergent series of complex terms for which $\sum\left|u_{k}\right|=+\infty$, and for this series let $\phi_{n}(\xi)\left[f_{n}(x)\right]$ be defined as in (1.5) $[(2.4)]$. We may consider the series of real and imaginary parts in the light of Theorem 2 [Theorem 3] and thus show that the set of all $\xi$ on $I[x$ in $D]$ for which we have $\lim \sup _{n}\left|\phi_{n}(\xi)\right|<\infty$ $\left[\lim \sup _{n}\left|f_{n}(x)\right|<\infty\right]$ is a set of the first category.

Michigan State College

\title{
A FORMULA FOR THE DIRECT PRODUCT OF CROSSED PRODUCT ALGEBRAS
}

\author{
SAUNDERS MACLANE AND O. F. G. SCHILLING
}

1. Introduction. In this note we wish to present a uniform treatment of certain properties of crossed products. A crossed product over any field $F$ is an algebra determined by a finite, separable, normal extension $N$ of $F$, with a Galois group $\Gamma$, and a certain factor $\operatorname{set}^{1} h$ of elements $h_{S, T}$ in $N$, for automorphisms $S$ and $T$ in $\Gamma$. The crossed product $(N, \Gamma, h)$ consists of all sums $\sum u_{S} z_{S}$, where the coefficients $z_{S}$ lie in $N$, and the fixed elements $u_{S}$ have the multiplication table

$$
u_{S} u_{T}=u_{S T} h_{S, T}, \quad z u_{S}=u_{S} z^{S}, \quad z \text { in } N .
$$

Let $K$ be a normal subfield of $N$, corresponding to the subgroup $\Delta$ of the Galois group $\Gamma$. A factor set $g$ in $N$ is called symmetric in $\Delta$ if $g_{S, T}=g_{U, V}$ whenever $S U^{-1}$ and $T V^{-1}$ are in $\Delta$.

Presented to the Society, May 3, 1941; received by the editors March 31, 1941.

${ }^{1}$ Definitions are given in A. A. Albert, Structure of Algebras, American Mathematical Society Colloquium Publications, vol. 24, 1939. Theorems cited below without explicit source all refer to this work. 
THEOREM 1. A crossed product $(N, \Gamma, \boldsymbol{h})$ is split by a normal subfield $K$ of $N$ if and only if its factor set $h$ is associate to a $\Delta$-symmetric factor set $\mathrm{g}$, where $\Delta$ is the subgroup of $\Gamma$ corresponding to $K$.

Part of this result may be stated more explicitly, using the factor group $\Gamma / \Delta$ as the Galois group of $K$ over $F$.

TheOREM 2. A factor set $g$ is $\Delta$-symmetric if and only if there is a factor set $G$ of elements $G_{\sigma, \tau}$ in $K$, where $\sigma, \tau$ are in $\Gamma / \Delta$, such that, for $S$ in the coset $\sigma, T$ in the coset $\tau$,

$$
g_{S, T}=G_{\sigma, \tau} .
$$

Furthermore the corresponding crossed products are similar,

$$
(N, \Gamma, g) \sim(K, \Gamma / \Delta, G) .
$$

An equivalent of these theorems was stated by Deuring. ${ }^{2}$ Since they were not used in Albert's Colloquium Lectures, Deuring's somewhat obscure proof is apparently the only one available. We give here a new proof. It is based on the simple observation that the standard proof ${ }^{3}$ of the formula

$$
(N, \Gamma, \boldsymbol{g}) \times(N, \Gamma, \boldsymbol{h}) \sim(N, \Gamma, \boldsymbol{g} h)
$$

can be extended to treat the case $(N, \Gamma, h) \times(K, \Gamma / \Delta, G)$. From this formula we obtain the theorems above, as well as a general formula for the direct product of two crossed products built on any two normal fields. In a systematic treatment, this proof has the advantage that it involves practically no more trouble than the proof of the ordinary product formula (4), and includes this as a special case.

2. Idempotents of matric subalgebras. It seems convenient to use the following restatement of known results about possible total matric subalgebras of a simple algebra.

TheOREm 3. Let the unity element of a simple algebra $A$ be represented as a sum $1=e_{1}+e_{2}+\cdots+e_{t}$ of pairwise orthogonal idempotents. Then $A$ has a total matric subalgebra $M$ with a basis $e_{i j}, i, j=1, \cdots, t$, having the usual multiplication table, $e_{i j} e_{j k}=e_{i k}, e_{i j} e_{m k}=0$ for $j \neq m$, and so constructed that $e_{i}=e_{i i}$, for $i=1, \cdots, t$, if and only if $A$ has for each $i$ an automorphism which maps $e_{1}$ on $e_{i}$.

${ }^{2}$ M. Deuring, Algebren, Ergebnisse der Mathematik und ihrer Grenzgebiete, vol. 4 , no. 1 (1935), pp. 62-64.

${ }^{3}$ Given in Albert, partly in Theorem 2.27 and partly in Theorem 5.6, and, originally, in somewhat different form, in $\mathrm{H}$. Hasse, Theory of cyclic algebras over an algebraic number field, Transactions of this Society, vol. 34 (1932), pp. 191-194. 
Proof. If $A$ contains $M$, then $A=M \times C$, where $C$ is the centralizer of $M$ (Theorem 1.17). The algebra $M$ has an inner automorphism mapping $e_{11}$ on $e_{i i}$, and this can be extended, so as to be the identity on $C$, to all of $A$. This proves the necessity of our condition.

Conversely, suppose each idempotent $e_{i}$ has the form $e^{\sigma}$, where $e=e_{1}, \sigma$ is a suitable automorphism. Decompose $e$ into primitive pairwise orthogonal idempotents of $A$ (Theorem 2.16), as $e=f_{1}+\cdots+f_{r}$. One then computes that the unity of $A$ can be decomposed into the pairwise orthogonal primitive idempotents $1=\sum f_{j}^{\sigma}$, where the sum is taken over all $j=1, \cdots, r$ and over all $\sigma$ needed to give the idempotents $e^{\sigma}$. The structure theorem (Theorem 3.19) then asserts that $A=M \times D$, where $M$ is a total matric algebra with basis $e_{i j}$, and where the diagonal elements $e_{k k}$ are the given idempotents $f_{j}^{\sigma}$. Each of the original idempotents $e_{i}$ is the sum of exactly $r$ idempotents $f_{j}^{\sigma}$. Hence in $M$ we select a subalgebra consisting of those matrices which are constructed from blocks of $r \times r$ scalar matrices. This subalgebra is itself a total matric algebra, and its idempotents are the given $e_{i}$.

3. The product formula. We now prove the following theorem:

THEOREM 4. If $K$ is a normal subfield of a normal field $N$, belonging to the subgroup $\Delta$, then the direct product of two crossed products to $N$ and $K$ is given by

$$
(N, \Gamma, h) \times(K, \Gamma / \Delta, G) \sim(N, \Gamma, h \xi),
$$

where $g$ is the $\Delta$-symmetric factor set obtained from the given set $G$ for $\Gamma / \Delta$ by the extension (2).

Proof. Let $C$ denote the direct product on the left of (5); since $C$ has a unity element, we may regard $F$ as a subfield of $C$. By the definition of a direct product, $C$ is generated by a subalgebra isomorphic to $(N, \Gamma, \boldsymbol{h})$, which we can identify with this algebra, and another subalgebra isomorphic to (but not identical with) the second factor $(K, \Gamma / \Delta, G)$; so we may write ${ }^{4} C$ as

$$
C=(N, \Gamma, h) \times\left(K^{\prime}, \Gamma / \Delta, G^{\prime}\right),
$$

where the subfields $K^{\prime}$ and $N$ have only the elements of $F$ in common, where $K$ is equivalent to $K^{\prime}$ over $F$ under a correspondence $y \leftrightarrow y^{\prime}$, and where $G^{\prime}$ is the map of $G$ under this isomorphism. Each coset of

${ }^{4}$ In (5), we simply use the ordinary convention, writing the direct product of two algebras not necessarily disjoint; in (6) we have $C$ represented more explicitly as the direct product of two of its subalgebras. The distinction is a familiar one. 
$\Gamma / \Delta$ may then be interpreted as an isomorphism of $K^{\prime}$ under the natural correspondence

$$
\left(y^{\prime}\right)^{\sigma}=\left(y^{S}\right)^{\prime}, \quad y \text { in } K, S \text { in the coset } \sigma .
$$

The crossed product $(N, \Gamma, h)$ is determined by the formulas (1), while $\left(K^{\prime}, \Gamma / \Delta, G^{\prime}\right)$ is determined by similar formulas

$$
v_{\sigma} v_{\tau}=v_{\sigma \tau} G_{\sigma, \tau}^{\prime} ; \quad y^{\prime} v_{\sigma}=v_{\sigma} y^{\prime \sigma}, \quad y \text { in } K .
$$

The automorphisms $S$ and $\sigma$ may be extended to the whole algebra $C$ of (6) by the formulas

$$
a^{S}=u_{S}^{-1} a u_{S}, \quad a^{\sigma}=v_{\sigma}^{-1} a v_{\sigma},
$$

for any $a$ in $C$. In a direct product, any term in one factor commutes with any term in the other, hence $S$ leaves fixed all elements of $K^{\prime}$ and $\sigma$ leaves fixed all elements of $N$.

The direct product $C$ contains the commutative subalgebra $N \times K^{\prime}$; since $N$ is separable, this algebra is semi-simple (chap. $3, \S 7$ ) and as such is the direct sum of fields $L_{i}$ with idempotents $e_{i}$. Since $1=\sum e_{i}$, $L_{i}$ has the form $e_{i}\left(N \times K^{\prime}\right)$. Let $L$ be one of these fields, with idempotent $e$. Then the mapping $z \rightarrow e z$ carries the elements $z$ of $N$ homomorphically into $L$; since both are fields, this must be an isomorphism of $N$ to part of $L$. For similar reasons, $y^{\prime} \rightarrow e y^{\prime}$ maps $K^{\prime}$ isomorphically on part of $L$. These two mappings agree on the common subfield $F$ of $N$ and $K^{\prime}$. Therefore $L$ contains the two fields $e K$ and $e K^{\prime}$, which are equivalent over $e F$ because $K$ and $K^{\prime}$ are equivalent over $F$. Since $K$ is normal over $F$, this implies that $e K=e K^{\prime}$. This identity means that for each element $y$ in $K$ there exists an element $y^{*}$ in $K^{\prime}$ such that $e y=e y^{*}$, and such that the mapping $y \rightarrow y^{*}$ is an equivalence of $K$ to $K^{\prime}$ over $F$. Now two mappings $y \rightarrow y^{\prime}$ and $y \rightarrow y^{*}$ of $K$ to $K^{\prime}$ can differ only by an automorphism $\sigma$ of $K^{\prime}$ over $F$, so that we may write $y^{\prime}=y^{* \sigma}$. One may then compute that the replacement of $e$ by the idempotent $e^{\sigma}$ simply replaces $y^{*}$ by $y^{\prime}$ in the equation $e y=e y^{*}$. Furthermore, $e^{\sigma}$ is the unity element of the field $L^{\sigma}$, which is a direct summand of $N \times K^{\prime}$ because $\sigma$, as defined by (8), is an automorphism of this algebra. Now change the notation, writing $L$ for $L^{\sigma}, e$ for $e^{\sigma}$; we then have in $N \times K^{\prime}$ a direct summand $L$ with unity $e$ such that

$$
e y=e y^{\prime},
$$

$y$ in $K$,

where $y \rightarrow y^{\prime}$ is the given equivalence of $K$ to $K^{\prime}$. Furthermore, $L=e N$, though we do not need this fact.

The idempotents $e^{\sigma}$, for $\sigma$ in $\Gamma / \Delta$, are all distinct. For suppose this were not the case; then $e^{\sigma}=e$ for some $\sigma \neq 1$, so that $e^{\sigma} y=e y=e y^{\prime}$ 
$=e^{\sigma} y^{\prime}$. On the other hand, one computes by (9) that

$$
e^{\sigma} y=v_{\sigma}^{-1} e v_{\sigma} y=v_{\sigma}^{-1}(e y) v_{\sigma}=v_{\sigma}^{-1}\left(e y^{\prime}\right) v_{\sigma}=e^{\sigma} y^{\prime \sigma},
$$

so that $e^{\sigma} y^{\prime}=e^{\sigma} y^{\prime \sigma}$. Since the correspondence $y^{\prime} \rightarrow e^{\sigma} y^{\prime}$ is one-one, this gives $y^{\prime}=y^{\prime \sigma}$ for all $y^{\prime}$, which means that $\sigma$ is the identity, contrary to assumption. The distinct idempotents $e^{\sigma}$ belong to $k$ distinct summands $L^{\sigma}$ of $N \times K^{\prime}$, where $k$ is the degree of $K$ over $F$. Since each summand $L^{\sigma}$ has at least the degree of $N$, these summands include all the direct summands of $N \times K^{\prime}$. Hence every primitive idempotent in $N \times K^{\prime}$ is one of the idempotents $e^{\sigma}$.

If $S$ is in the coset $\sigma$, then $\left(e^{S}\right)^{\sigma}=e$; for one may compute the effect of multiplying $\left(e^{S}\right)^{\sigma}$ by an element $x^{S}$ of $K$, getting

$$
e^{S \sigma} x^{S}=e^{S \sigma} x^{S \sigma}=(e x)^{S \sigma}=\left(e x^{\prime}\right)^{S \sigma}=e^{S \sigma} x^{\prime S \sigma}=e^{S \sigma} x^{\prime \sigma}=e^{S \sigma}\left(x^{S}\right)^{\prime},
$$

where the last transformation uses the definition (7) of the automorphism $\sigma$. Since any element $y$ of $K$ can be written in the form $y=x^{S}$, this proves that $e^{S \sigma} y=e^{S \sigma} y^{\prime}$, for every $y$. On the other hand, $e^{S \sigma}$ is a primitive idempotent, hence is $e^{\tau}$ for some $\tau$ in $\Gamma / \Delta$. As in (10), one then computes that $e^{S \sigma} y=e^{\tau} y=e^{\tau} y^{\prime \tau}$. Compared with the previous equation, this means that $y^{\prime r}=y^{\prime}$, hence that $\tau=1$, hence that $e^{S \sigma}=e$, as asserted.

The conclusion $e^{S \sigma}=e$ may be reinterpreted in terms of the definitions (8) of the extended automorphisms $S$ and $\sigma$. It then becomes the assertion that $e$ commutes with the product $u_{S} v_{\sigma}$. If $S$ is in the coset $\sigma$, we write $w_{S}$ for $u_{S} v_{\sigma}$, and have

$$
e w_{S}=w_{S} e, \quad w_{S}=u_{S} v_{\sigma} .
$$

The idempotents $e, e^{\sigma}, e^{\tau}, \cdots$ of $N \times K^{\prime}$ are all conjugate in the given algebra $C$ of (6); hence Theorem 3 provides a total matric subalgebra $M$ of $C$ of degree $k$ and with basis $e_{i j}$, where $e_{11}=e, e_{22}=e^{\sigma}, \cdots$. This algebra is a direct factor of $C$ (Theorem 1.17); so

$$
C=(N, \Gamma, h) \times\left(K^{\prime}, \Gamma / \Delta, G\right)=M \times B,
$$

where $B$ is the $C$-centralizer of $M$. By the structure of a total matric algebra $e C e$ will be a subalgebra equivalent to $B$. This subalgebra contains a subfield $e N e=e N$ isomorphic to $N$, with automorphisms $e z \leftrightarrow e z^{S}$, and also contains elements $e w_{S} e=e w_{S}$ of (11), one for each automorphism. The multiplication table for these elements may be computed, using the fact that $e G=e G^{\prime}$; it is

$$
(e z)\left(e w_{S}\right)=\left(e w_{S}\right)\left(e z^{S}\right), \quad\left(e w_{S}\right)\left(e w_{T}\right)=\left(e w_{S T}\right)\left(e h_{S, T} G_{\sigma, \tau}\right),
$$

where $S$ and $T$ lie respectively in the cosets $\sigma$ and $\tau$. Since the whole 
algebra $e C e$ has the same degree as $N$, this means simply that $e C e$ is a crossed product to $e N$ and the factor set $e h_{S, T} G_{\sigma, \tau}$ (Albert, p. 67). Therefore (12) proves that $C$ is similar to a crossed product $B$ of the desired form.

4. Properties of symmetric factor sets. We now return to the proof of Theorem 2 of the introduction. Given a factor set $G$ of elements in $K$, one proves at once that the definition (2) for $\boldsymbol{g}$ does yield a factor set for $\Gamma$. Conversely, if a given factor set $g$ is $\Delta$-symmetric, the associativity conditions $g_{S, T R} g_{T, R}=g_{S T, R}\left(g_{S, T}\right)^{R}$ for $R$ in $\Delta$ become

$$
g_{S, T} g_{T, 1}=g_{S T, 1}\left(g_{S, T}\right)^{R} .
$$

But the associativity conditions with $R=1$ make $g_{T, 1}=g_{S T, 1}$, so the result above becomes $\left(g_{S, T}\right)^{R}=g_{S, T}$; hence each element $g_{S, T}$ of the factor set lies in the subfield $K$. One may then define $G_{\sigma, \tau}$ by (2), and show that $G$ is a factor set for $K$. For $G$ so defined, the formula of Theorem 4 gives

$$
\left(N, \Gamma, \boldsymbol{g}^{-1}\right) \times(K, \Gamma / \Delta, G) \sim\left(N, \Gamma, \boldsymbol{g}^{-1} \boldsymbol{g}\right) \sim(N, \Gamma, 1) \sim 1 .
$$

Multiplying by $(N, \Gamma, \boldsymbol{g})$, one concludes that $(K, \Gamma / \Delta, G) \sim(N, \Gamma, \boldsymbol{\xi})$, as in the formula (3) of Theorem 2.

Theorem 1 now follows formally from Theorem 2. For, if an algebra $(N, \Gamma, \boldsymbol{h})$ is split by the normal subfield $K$, it is $\operatorname{similar}^{5}$ to a crossed product $(K, \Gamma / \Delta, G)$, and by Theorem 2 the latter algebra is in turn similar to $(N, \Gamma, \boldsymbol{g})$, where $\boldsymbol{g}$ is the $\Delta$-symmetric extension of $G$. But $(N, \Gamma, \boldsymbol{h}) \sim(N, \Gamma, \boldsymbol{g})$ gives $\boldsymbol{h} \sim \boldsymbol{g}$ (Theorem 5.5$)$, so $\boldsymbol{h}$ is the associate of a $\Delta$-symmetric factor set $g$, as asserted. Conversely, if $h$ is associate to a $\Delta$-symmetric factor set $g$, then

$$
(N, \Gamma, h) \sim(N, \Gamma, \boldsymbol{g}) \sim(K, \Gamma / \Delta, G),
$$

and the latter algebra is indeed split by $K$. This completes the proof of Theorem 1.

5. Arbitrary direct products. Now we consider two crossed products to any two given fields $K$ and $K^{\prime}$ which are finite, separable, and normal over a common base field $F$. The composite $N=K \cup K^{\prime}$ of $K$ and $K^{\prime}$ is uniquely determined; we may regard $K$ and $K^{\prime}$ as subfields of $N$. The Galois group $\Gamma$ of $N / F$ is determined in terms of the groups $\Sigma$ and $\Sigma^{\prime}$ of $K / F$ and $K^{\prime} / F$ as follows. ${ }^{6}$

${ }^{5}$ Because any normal simple algebra $A$ split by a field $K$ normal over $F$ is similar to a crossed product to this field $K$. This well known fact is contained in the proof of Theorem 5.1.

${ }^{6}$ This result is known, although explicit citations are rare. To prove it, observe that any $S$ induces and is determined by $\sigma$ and $\sigma^{\prime}$, and then count the number of al- 
LEMma. Let a field $N$ be the join of two subfields $K$ and $K^{\prime}$, each finite, separable, and normal over a common base field $F$, so that $N$ is also finite, separable, and normal over $F$. If $\sigma, \sigma^{\prime}$ are automorphisms of $K, K^{\prime}$, respectively, which have the same effect on each element of the intersection $K \cap K^{\prime}$, then there exists one and only one automorphism $S$ of $N / F$ which induces the given automorphisms $\sigma$ and $\sigma^{\prime}$. Every automorphism $S$ of $N / F$ may be obtained in this way, and the correspondence $S \leftrightarrow\left(\sigma, \sigma^{\prime}\right)$ maps the Galois group of $N / F$ isomorphically on a subgroup of the direct product of the Galois groups of $K / F$ and $K^{\prime} / F$.

Our most inclusive result on direct products now is the following theorem:

Theorem 5. Let $(K, \Sigma, G)$ and $\left(K^{\prime}, \Sigma^{\prime}, G^{\prime}\right)$ be any two given crossed products to fields $K$ and $K^{\prime}$ normal over $F$. Let $S \leftrightarrow\left(\sigma, \sigma^{\prime}\right)$ and $T \leftrightarrow\left(\tau, \tau^{\prime}\right)$ be any two automorphisms of the composite field, determined, as in the lemma, in terms of automorphisms of $K$ and $K^{\prime}$, and extend the given factor sets $G$ and $G^{\prime}$ to factor sets for $K \cup K^{\prime}$ by the formulas

$$
g_{S, T}=G_{\sigma, \tau}, \quad g_{S, T}^{\prime}=G_{\sigma^{\prime}, \tau^{\prime}}^{\prime}, \quad S \rightarrow\left(\sigma, \sigma^{\prime}\right), \quad T \rightarrow\left(\tau, \tau^{\prime}\right) .
$$

Then the direct product of the two given crossed products is

$$
(K, \Sigma, G) \times\left(K^{\prime}, \Sigma^{\prime}, G^{\prime}\right) \sim\left(K \cup K^{\prime}, \Gamma, g \boldsymbol{g}^{\prime}\right) .
$$

In the special case when $K$ and $K^{\prime}$ are disjoint, the Galois group of $K \cup K^{\prime}$ is just the direct product of the two groups $\Sigma$ and $\Sigma^{\prime}$ and the formulas (13) mean simply that the matrix of $\mathbf{g}^{\prime}$ is the Kronecker product of the matrices $G$ and $G^{\prime}$. This case has already been considered by one of us. ${ }^{7}$ In the case when $K$ is a subfield of $K^{\prime}$, the formula (14) specializes to the formula derived in Theorem 4. This special case gives a proof of (14) in general, for observe that $\left(\sigma, \sigma^{\prime}\right) \rightarrow \sigma$ maps $\Gamma$ homomorphically on $\Sigma$, so that the formula (13) really extends $G$ to be a factor set for $\Gamma$ which is symmetric relative to a suitable subgroup $\Delta$. Therefore $(K, \Sigma, G) \sim\left(K \cup K^{\prime}, \Gamma, \xi\right)$ by (3). The analogous result for $K^{\prime}$ then gives (14).

HARVARD UNIVERSITY AND

The University of Chicago

lowable pairs $\left(\sigma, \sigma^{\prime}\right)$. If $K, K^{\prime}$ and $K \cap K^{\prime}$ have over $F$ the respective degrees $k, k^{\prime}$, and $d$, then the degree of $K \cup K^{\prime}$ over $F$ is $k k^{\prime} / d$, and the number of pairs $\left(\sigma, \sigma^{\prime}\right)$ which agree on the intersection $K \cap K^{\prime}$ is also $k k^{\prime} / d$. Hence every $\left(\sigma, \sigma^{\prime}\right)$ is realized as an automorphism $S$ of $K \cup K^{\prime}$.

${ }^{7}$ O. F. G. Schilling, The structure of certain rational infinite algebras, Duke Mathematical Journal, vol. 3 (1937), p. 305. 